\title{
Estruturação de um Serviço de Informações sobre Medicamentos (SIM) em um hospital do Rio Grande do Norte, Brazil
}

\author{
Structuring a Drug Information Services (DIS) in a \\ hospital at Rio Grande do Norte State, Brazil
}

Recebido em: 01/03/2019 Aceito em: $\quad 05 / 06 / 2019$

\author{
Álex Brunno Nascimento MARTINS ${ }^{1}$; Almária Mariz BATISTA ${ }^{2}$ \\ ${ }^{1}$ Escola Multicampi de Ciências Médicas, Universidade Federal do \\ Rio Grande do Norte. Rua Dr. Carlindo Dantas, s/n, CEP 59300-000. \\ Caicó, RN. Brasil. ${ }^{2}$ Departamento de Farmácia, Centro de Ciências da Saúde, \\ Universidade Federal do Rio Grande do Norte. Av. General Gustavo \\ Cordeiro de Farias, s/n, CEP 59010-180. Natal, RN. Brasil. \\ E-mail: almariamariz@yahoo.com.br
}

\section{ABSTRACT}

Drug Information Centers/Drug Information Services are important and useful tools for information related to drugs. The information also is actively disclosed to the population and may contribute to the rational use of drugs. In 2017, a Drug Information Service was implanted at Hospital Regional Dr Mariano Coelho in Currais Novos, RN. This study aimed to evaluate the information provided by this Service after 19 months of operation. Therefore, a cross-sectional, descriptive and exploratory study was carried out on the Service database, whose information requests were received between April 2017 and November 2018. The information was evaluated from a monthly average of information requests, type of requests, receive mode requests, the profile of the enquirer, information sources utilized to answer to requests and the pharmacological classification of the drugs involved. During the period, 31 requests were received (1.6 requests/ month). The majority of them had how the type of request indication of use of drugs $(22.6 \%)$, were personally received (71.0\%) and were requested by multi-professional residents (45.2\%). Electronic databases were the most utilized sources to answer to requests (40.0\%), and antibiotics of systemic use were the most drugs present in the requests $(23.7 \%)$. It appears this Service was potentially useful to avoid the irrational use of drugs. Regarding the requested profile of other centres/services, this showed a profile little similar to the others. An additional evaluation of this Service considering other parameters is necessary.

Keywords: Pharmaceutical Assistance; drug information services; hospitals

\section{RESUMO}

Centros/Serviços de Informações sobre Medicamentos são importantes e úteis, pois contribuem para o uso racional de medicamentos. O objetivo deste estudo foi avaliar as informações prestadas pelo Serviço de Informações sobre Medicamentos do Hospital Regional Dr. Mariano Coelho em Currais Novos, RN. Para tanto, foi realizado um estudo observacional a partir do banco de dados do Serviço, cujas solicitações de informação foram recebidas entre abril de 2017 e novembro de 2018. As informações foram avaliadas a partir de média mensal de solicitações de informação, temas das perguntas, meio pelo qual foi recebida, perfil do solicitante, fontes de informação utilizadas e classificação farmacológica dos medicamentos en- 
volvidos. Durante o período, foram recebidas 31 solicitações (1,6 solicitações/mês), a maioria tinha como tema a indicação de uso de medicamentos $(22,6 \%)$ e foi solicitada pessoalmente $(71,0 \%)$ por residentes multiprofissionais $(45,2 \%)$. Bases de dados eletrônicas foram as fontes de informações mais utilizadas para responder às solicitações (40,0\%); e os antibióticos de uso sistêmico os medicamentos mais presentes nas solicitações $(23,7 \%)$. Depreende-se que este Serviço foi potencialmente útil para se evitar uso irracional de medicamentos. Em relação ao perfil de solicitações de outros centros/serviços, este apresentou perfil pouco semelhante aos demais.

Palavras-chave: Assistência Farmacêutica; serviços de informação sobre medicamentos; hospitais

\section{INTRODUÇÃO}

Após a Segunda Guerra Mundial, o mundo experimentou grandes inovações decorrentes do grande desenvolvimento industrial. Uma das indústrias que muito se fortaleceu foi a farmacêutica que, nas décadas seguintes, disponibilizou ao mercado uma grande quantidade de novos medicamentos elevando, a uma diversidade de opções, a terapêutica medicamentosa (1).

À medida que esses medicamentos eram disponibilizados, aumentavam as publicações científicas sobre eles, o que, por sua vez, contribuía para o aumento de questionamentos acerca do interesse e da qualidade das produções. Isto demandava um profissional que pudesse disponibilizar, com imparcialidade e precisão, informações sobre os medicamentos que acabavam de surgir. Este profissional era o farmacêutico (2).

Dessa forma, nos dias de hoje, o farmacêutico continua a se colocar no contexto de prestador de informações seguras e confiáveis em uma época em que a informação é tão democrática devido à internet. $\mathrm{O}$ farmacêutico, neste novo contexto, ganha o papel de guia e intérprete da informação inserida e disseminada na rede mundial (3).

Segundo a Organização Mundial da Saúde (OMS), os conhecimentos especializados do farmacêutico sobre a conduta da terapêutica medicamentosa e sobre as propriedades dos medicamentos, num ambiente de atenção à saúde cada vez mais sofisticado, aproxima-o do prescritor, como fonte independente de informações acerca de opções terapêuticas e consequências, positivas e negativas, do tratamento. Também os aproxima dos pacientes na comunidade, como dispensadores acessíveis não apenas de medicamentos, mas também de informações sobre a saúde (4).
Um dos meios de prestação de informações por farmacêutico é o Centro/Serviço de Informações sobre Medicamentos (CIM/SIM), que constitui um local onde informações sobre medicamentos são avaliadas, divulgadas, analisadas e reunidas. $\mathrm{O}$ primeiro CIM foi criado em 1962, no Centro Médico da Universidade de Kentucky, EUA. Desde então, outros CIM/SIM foram sendo criados em vários países, sendo o primeiro CIM brasileiro implantado no Hospital Universitário Onofre Lopes, antigo Hospital das Clínicas, em Natal, RN $(5,6)$.

Em 1994, foi criado o Centro Brasileiro de Informação sobre Medicamentos (CEBRIM) vinculado ao Conselho Federal de Farmácia (CFF), com o apoio da Organização Pan-Americana da Saúde (OPAS) (2). Em 1999, um protocolo de cooperação estabeleceu os critérios para instauração de novos Centros no Brasil e integrou os já existentes ao Sistema Brasileiro de Informação sobre Medicamentos (SISMED). Em 2010, com o apoio do Ministério da Saúde e da OPAS, foi criada a Rede Brasileira de Centros e Serviços de Informação sobre Medicamentos (REBRACIM) que, 3 anos depois, foi institucionalizada no Sistema Único de Saúde por meio da Portaria $n^{\circ}$ 2.647/13 (7), integrando, à época, 24 Centros em 10 Estados e no Distrito Federal (8).

É necessário diferenciar um CIM de um SIM. $\mathrm{O}$ primeiro está relacionado à estrutura física. $\mathrm{O}$ segundo, à atividade desempenhada. Adicionalmente, foi adotada uma classificação que denota hierarquia ao convencionar que Centros têm grande abrangência, como o País ou um Estado. Serviços estão restritos a instituições (6).

Um CIM/SIM pode ser instalado em diferentes instituições como hospitais, indústrias ou universidades, sendo prestadas informações passivas e ativas. Informação passiva responde a uma soli- 
citação, que pode ser um questionamento por um usuário acerca de farmacoterapia ou medicamento específico. Informação ativa se dá por atividades de educação e consultoria sobre medicamentos e saúde $(2,9)$. Em pesquisa com alguns CIM/SIM brasileiros, foi estimado que a informação passiva corresponde a até $80 \%$ das atividades de $88 \%$ de todos eles (10).

No Hospital Regional Mariano Coelho, em Currais Novos, RN, em abril de 2017 foi implantado um SIM como forma de promover o uso racional de medicamentos. Durante 19 meses, os farmacêuticos prestaram informações a profissionais do hospital, atendendo solicitações a respeito de diferentes temas.

Diante desse contexto, o objetivo desse estudo foi avaliar as informações prestadas pelo SIM, expondo a capacidade do farmacêutico em prestar informações pertinentes, de qualidade e independentes sobre medicamentos.

A importância deste estudo justifica-se pela necessidade de se expor a iniciativa do farmacêutico em desenvolver o Serviço, de contribuir com a discussão dos Centros e Serviços de Informações sobre medicamentos no Brasil e de aprimorar a atuação deste Serviço na Instituição.

\section{MÉTODO}

Trata-se de estudo observacional, a partir do banco de dados de um SIM, cujas solicitações de informação foram recebidas de abril de 2017 a novembro de 2018.

Por não envolver diretamente seres humanos como alvo desta pesquisa, não foi necessário submetê-la a Comitê de Ética em Pesquisa.

O SIM está localizado no Hospital Regional Mariano Coelho, na cidade de Currais Novos, RN. Trata-se de hospital geral administrado pela esfera estadual, referência para Região do Seridó Potiguar. O hospital tem 88 leitos distribuídos entre os setores de clínicas médica, cirúrgica, pediátrica e obstétrica (alojamento conjunto), além de unidade de terapia intensiva (UTI) (adulto) e unidade de cuidados intermediários (UCI) (neonatal). O alojamento conjunto é o setor que recebe o binômio mãe-filho logo após o parto. Sua capacidade é de
15 leitos. O Hospital, em parceria com a Universidade Federal do Rio Grande do Norte, constitui campo de prática da Residência Multiprofissional em Saúde Materno-Infantil.

Para atendimento das solicitações de informação (SI), o SIM disponibilizou número de telefone e endereço de e-mail. Assim, estas solicitações podem ser recebidas por aplicativo de celular, correio eletrônico ou pessoalmente pelos farmacêuticos. Tanto as SI quanto as repostas a estas são inseridas em documento do Microsoft Word $\AA 2$ 2013, segundo formulário próprio para respostas, de forma que as respostas às SI são impressas em duas vias, sendo uma enviada ao solicitante e outra arquivada em pasta que alimenta o banco de dados do Serviço. A depender da possibilidade de se entregar, pessoalmente, a resposta à SI, esta é arquivada em formato .pdf e enviada via o aplicativo WhatsApp.

As respostas às SI foram elaboradas por dois farmacêuticos da Residência Multiprofissional. Para tal, foram consultadas fontes de informação primárias, secundárias e terciárias e as respostas inseridas em formulário com identificação do solicitante, informação solicitada, dados do paciente, código de resposta à solicitação, conclusão resumida, referências e campo para assinatura do consultor.

As SI foram avaliadas a partir de média mensal de solicitações de informação, caracterização dos temas das perguntas, meio pelo qual foi recebida, perfil do solicitante, fontes de informação utilizadas e classificação farmacológica dos medicamentos envolvidos, via Anatomical Therapeutic Chemical Code (ATC) (11). Nesse sistema, os medicamentos, conforme seu local de ação e propriedades farmacológicas e químicas, são classificados em diferentes grupos e subgrupos gerando códigos. Há 5 níveis de classificação ( $1^{\circ}$ ao $5^{\circ}$ nível). À medida em que se avança nos níveis, a especificação aumenta com a inclusão de novos números e letras; assim, ao chegar ao $5^{\circ}$ nível tem-se um código formado que representa um medicamento específico. Neste estudo a classificação abrangeu até o $2^{\circ}$ nível pois foi o suficiente para categorizar os medicamentos.

A análise dos dados foi realizada por meio do banco de dados do SIM a partir do software Microsoft Excel, a partir da tabulação em pla- 
nilha da caracterização das SI conforme os parâmetros já mencionados. O acesso ao banco de dados para coleta foi autorizado pelo diretor do Hospital, mediante carta de anuência para realização da pesquisa.

\section{RESULTADOS E DISCUSSÃO}

Durante o período, foram recebidas 31 SI, uma média de 1,6 solicitações mensais. Dentre estas, 15 (48\%) estavam diretamente relacionadas a pacientes.

Quanto à temática das SI, foram identificados 9 temas (Tabela 1). Algumas SI continham mais de uma pergunta, resultando em enquadramento em mais de um tema.

Com relação aos temas das SI, a maioria estava relacionado à indicação de uso $(22,6 \%)$, seguida de reação adversa/contraindicação $(19,4 \%)$, interações/incompatibilidades $(16,1 \%)$ e apresentação de medicamentos $(12,9 \%)$.

Em relação aos meios de recebimento das SI, a maior parte foi recebida pessoalmente pelos consultores $(71 \%)$ e as demais via contato de WhatsApp disponibilizado (29\%). Nenhuma SI foi recebida via e-mail. Cada SI foi recebida exclusivamente por um meio dos meios recebimento, sem duplicação.

Tabela1. Caracterização das solicitações de informação (SI)sobre medicamentos atendidas pelo Serviço de Informação sobre Medicamentos (SIM) do Hospital Regional Mariano Coelho, Currais Novos, RN, no período de abril de 2017 a novembro de 2018.

\begin{tabular}{|c|c|}
\hline \multicolumn{2}{|c|}{ TEMÁTICA DAS SOLICITAÇÕES } \\
\hline Indicação de uso & $22,6 \%$ \\
\hline Reações adversas/contraindicação & $19,4 \%$ \\
\hline Interações/incompatibilidades medicamentosas & $16,1 \%$ \\
\hline Apresentação de medicamentos & $12,9 \%$ \\
\hline Posologia/dose de medicamentos & $9,7 \%$ \\
\hline Conservação/estabilidade de medicamentos & $9,7 \%$ \\
\hline Preparo/administração de medicamentos & $9,7 \%$ \\
\hline Comparação entre medicamentos & $6,5 \%$ \\
\hline 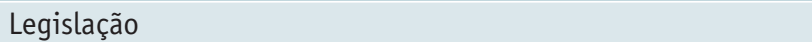 & $3,2 \%$ \\
\hline \multicolumn{2}{|c|}{ MEIO DE RECEBIMENTO DAS SOLICITAÇÕES } \\
\hline Pessoalmente & $71,0 \%$ \\
\hline Aplicativo de mensagens instantâneas para celular (WhatsApp) & $29,0 \%$ \\
\hline \multicolumn{2}{|c|}{$\begin{array}{ll}\text { PERFIL DO SOLICITANTE } \\
\end{array}$} \\
\hline \multicolumn{2}{|l|}{ Residentes multiprofissionais $(45,2 \%)$} \\
\hline Psicólogo & $16,1 \%$ \\
\hline Nutricionista & $12,9 \%$ \\
\hline Fisioterapeuta & $9,7 \%$ \\
\hline Enfermeiro & $6,5 \%$ \\
\hline \multicolumn{2}{|l|}{ Profissionais lotados no serviço $(35,5 \%)$} \\
\hline Técnico de enfermagem & $19,4 \%$ \\
\hline Psicólogo & $12,9 \%$ \\
\hline Médico & $3,2 \%$ \\
\hline Paciente & $9,7 \%$ \\
\hline Estudante de Medicina & $6,5 \%$ \\
\hline Desconhecido & $3,2 \%$ \\
\hline \multicolumn{2}{|c|}{ FONTES DE CONSULTA UTILIZADAS } \\
\hline Bases de dados eletrônicas & $40,0 \%$ \\
\hline Bulário & $25,0 \%$ \\
\hline Artigos científicos & $17,5 \%$ \\
\hline Outros sites & $10,0 \%$ \\
\hline Livros & $7,5 \%$ \\
\hline
\end{tabular}




\begin{tabular}{|l|r|}
\hline J01 - Antibacterianos de uso sistêmico & $23,7 \%$ \\
\hline M01 - Anti-inflamatórios e antirreumáticos & $18,4 \%$ \\
\hline A02 - Medicamentos relacionados a desordens ácidas & $7,9 \%$ \\
\hline A06 - Medicamentos para constipação & $7,9 \%$ \\
\hline A11 - Vitaminas & $7,9 \%$ \\
\hline B03 - Preparações antianêmicas & $5,3 \%$ \\
\hline N02 - Analgésicos & $5,3 \%$ \\
\hline A03 - Medicamentos para desordem funcional do trato gastrointestinal & $2,6 \%$ \\
\hline A10 - Medicamentos utilizados no diabetes & $2,6 \%$ \\
\hline B05 - Substitutos sanguíneos e soluções para perfusão & $2,6 \%$ \\
\hline D03 - Preparações para o tratamento de feridas e úlceras & $2,6 \%$ \\
\hline G04 - Urológicos & $2,6 \%$ \\
\hline H02 - Corticosteroides de uso sistêmico & $2,6 \%$ \\
\hline J02 - Antimicóticos de uso sistêmico & $2,6 \%$ \\
\hline N06 - Psicoanalépticos & $2,6 \%$ \\
\hline R06 - Antihistamínicos de uso sistêmico & $2,6 \%$ \\
\hline
\end{tabular}

Sobre o perfil dos solicitantes, $45,2 \%$ eram residentes multiprofissionais e $35,5 \%$ profissionais lotados no serviço. Quanto aos residentes, psicó$\log 0$ (16,1\%) e nutricionistas $(12,9 \%)$ foram a maioria. Quanto aos profissionais lotados no serviço, técnicos de enfermagem $(19,4 \%)$ e psicólogos $(12,9 \%)$ foram os que mais solicitaram.

No que se refere às fontes de informação, quase sempre foram utilizadas mais de uma fonte diferente para responder a uma única SI. As bases de dados eletrônicas foram as mais utilizadas (40\%), seguidas de bulas de medicamentos (25\%).

A maioria das SI citava diretamente ao menos um medicamento, predominando os antibacterianos de uso sistêmico $(23,7 \%)$, seguidos de anti-inflamatórios e antirreumáticos (18,4\%). Medicamentos relacionados a desordens gástricas, medicamentos para constipação e vitaminas corresponderam a 7,9\% das SI cada.

Em relação ao aproveitamento do Serviço pelo público, quando é comparado com outros (12-18), pode ser constatado que, considerando a média de recebimento de SI (1,6/mês), o SIM objeto de análise foi relativamente pouco solicitado, o que pode ser explicado pelo reduzido tamanho do Hospital, que dispõe de menos leitos, menos profissionais e, assim, menor possibilidade de questionamentos. $\mathrm{O}$ pouco tempo de funcionamento do SIM e o pouco conhecimento sobre sua existência pelos profissionais pode também explicar a baixa demanda.
As questões supracitadas também foram cogitadas como justificativa à baixa demanda de SI (1,1/mês) recebida por CIM de um instituto de pós-graduação indiano (19). Em um CIM da Etiópia também foi constatada uma baixa demanda de SI (0,9/mês), sendo apresentada, como possível causa, a falta de registro de todas as SI, o que costuma acontecer quando são realizadas solicitações pessoalmente em meio a rounds (20). O SIM em estudo não compartilhou desta questão, uma vez que todas as SI foram corretamente registradas.

Foi realizada a divulgação do Serviço pelos 2 farmacêuticos consultores durante as primeiras semanas de atividade. A divulgação consistiu, primeiramente, em um momento de apresentação do Serviço aos residentes multiprofissionais do Hospital e, depois, aos demais profissionais da Instituição em seus setores. Além da apresentação verbal, houve distribuição de panfletos via canais de envio de SI.

A temática mais frequente de SI, indicação de medicamentos, revela que as perguntas não envolviam elevado grau de complexidade de resposta (pelo menos em nível farmacêutico). $\mathrm{O}$ fato de a maioria dos solicitantes não contar com Farmacologia em suas formações pode ter gerado esse perfil de tema. A partir de comparação com outros estudos, foi observado um perfil semelhante apenas em um estudo desenvolvido em hospital terciário na Índia (14).

A maioria das SI foi recebida pessoalmente pelo SIM. Os farmacêuticos deste Serviço desenvolviam concomitantemente serviços clínicos nos 
leitos do hospital em meio à equipe multiprofissional. Este contato entre farmacêutico e demais profissionais facilita o recebimento de SI pessoalmente (16). Outrossim, a disponibilidade via WhatsApp demonstrou a proposta deste SIM em ser acessível, incorporando meios mais práticos e presentes no dia-a-dia. Não foram encontrados outros estudos que relatavam este canal para recebimento de SI. Pode ser que ainda não tenha havido tempo hábil para publicação de estudos com esta ferramenta, que é relativamente nova.

Foi feita a opção, inicialmente, pela não padronização do formulário de SI, para tornar o SIM mais acessível. No entanto, foi constatado o recebimento de várias perguntas abertas, ausentes de detalhes importantes. Então, foi concluído que isto compromete o desenvolvimento da resposta. Para não afetar a qualidade do serviço e contornar o problema, em curto prazo, foram solicitados detalhes necessários ao solicitante e, em algumas situações, as SI foram respondidas conjecturando diferentes possibilidades. Em longo prazo, está sendo desenvolvido formulário próprio para SI.

Em 2017, pesquisadores analisaram 27 CIM/ SIM integrados à REBRACIM. Eles os acionaram via envio de SI e avaliaram as respostas recebidas sob vários aspectos. No que se refere a formulários, constataram que 21 deles também não exigem formulários padronizados para SI (8), o que corrobora os resultados deste estudo. No mesmo estudo, no que tange às respostas, só em $45 \%$ deles foi constatada resposta em arquivo com extensão pdf, constando código de controle da SI. Os pesquisadores justificaram a necessidade dos 2 itens: dificulta possíveis alterações no texto e facilita consultas futuras. Os outros $55 \%$ da referida pesquisa enviavam respostas em texto livre no próprio corpo do e-mail, sem seguir padronização (8).

Em relação aos formulários para resposta, estes foram padronizados pelo SIM em análise. Este SIM em estudo atendeu aos 2 quesitos.

Quanto ao perfil do solicitante da informação, nem todo o público que utilizou o CIM/SIM era composto por profissionais do hospital. Pacientes também chegaram a consultar, ainda que em menor frequência. Isso demonstra a disponibilidade do SIM em sanar dúvidas de um público abrangente, inclusive leigos.
Os psicólogos, apesar de não lidarem diretamente com medicamento, tiveram posição de destaque como profissionais (residentes ou lotados no serviço) que mais acionaram o SIM, em que predominaram SI relacionadas à indicação de uso. $\mathrm{O}$ que pode explicar o fato é a incipiente abordagem da Farmacologia na formação de tais profissionais, o que contribui para que haja mais dúvidas de sua parte por ocasião de atuação junto ao paciente; consequentemente, há necessidade de apoio adequado para sanar dificuldades. $\mathrm{O}$ destaque destes profissionais também pode ressaltar atuação direta no cuidado ao paciente e a interação com a equipe multiprofissional. Não foi constatado outro CIM/ SIM que apresentasse tamanho destaque desta categoria profissional nas SI.

Técnicos de enfermagem também solicitaram informações em quantidade expressiva, o que era esperado pelo fato de investirem tempo considerável em preparo e administração de medicamentos. Foram solicitadas, principalmente, informações acerca de conservação e estabilidade, imprescindíveis para sua atividade.

Cabe ainda destacar a falta de SI por parte de farmacêuticos neste SIM, categoria que costuma figurar entre as que mais solicitam informações via $\operatorname{CIM} / \operatorname{SIM}(3,13,17,18)$. O reduzido número de farmacêuticos lotados no serviço, aliado à carência de serviços clínicos por eles desenvolvidos, foram determinantes para ausência de SI.

Sobre as fontes consultadas para resposta às SI, na maioria das vezes, foram utilizadas bases de dados eletrônicas (Uptodate e Micromedex). Isto qualifica as respostas, uma vez que tais fontes, são reconhecidas como independentes e provedoras de informações prontamente atualizadas. $\mathrm{O}$ uso de tais fontes se repeteu em outros CIM/SIM. Em um hospital terciário indiano, foi utilizada, na maioria das vezes, especificamente, Micromedex (16).

Às vezes, foram necessários dados mais específicos sobre medicamentos, os quais podem variar de fabricante para fabricante como o tempo de estabilidade após a reconstituição e condições de armazenamento; por isso, também foi consideravelmente utilizado o bulário próprio, construído a partir da reunião de bulas integrantes dos medicamentos que o Hospital adquiriu ao longo do tempo. O bulário 
da Agência Nacional de Vigilância Sanitária (Anvisa) (21) disponível via Internet, quando da indisponibilidade da bula em bulário próprio, também foi utilizado.

A categoria "outros sites" está relacionada à busca em sites oficiais de fabricantes de medicamentos e de veiculação de legislação brasileira. A necessidade de recorrer a tais sites foi devida à demanda por comunicados oficiais da indústria farmacêutica e normas brasileiras para responder a algumas SI.

A respeito dos medicamentos mais prevalentes nas SI, antibacterianos de uso sistêmico podem ter recebido destaque devido à considerável frequência de uso na instituição. Não há estudos de perfil de uso de medicamentos no Hospital, no entanto, foi observado um considerável uso de antimicrobianos. Esta classe também figurou entre as mais presentes em SI da Etiópia (20) e da Malásia (22).

O subgrupo anti-inflamatórios e antirreumáticos pode ter ganhado destaque devido à razoável frequência de uso destes medicamentos no Hospital, bem como o fato de que parte das SI neste subgrupo estava relacionada a uso de medicamentos no cenário de problemas de saúde autolimitados, cujos medicamentos eram isentos de prescrição, e não necessariamente necessitavam de profissional que os prescrevesse para que a aquisição dos mesmos fosse efetivada. Assim, gerou dúvidas aos usuários, que acabaram questionando ao SIM acerca do uso correto.

Revisando a literatura acerca de estudos com foco na atividade de Centros e Serviços ao redor do mundo, foram constatados itens não avaliados neste estudo, como índice de satisfação dos usuários, avaliação de consultores externos (sobre as respostas), tempo de resposta, dados demográficos dos solicitantes, horário de maior recebimento de
SI e propósito da SI $(14,16,23,24)$. Também não foi avaliado o impacto das respostas às SI sobre o benefício ao usuário, o que gera a perspectiva de estudos futuros acerca destas questões.

Os resultados constatados neste estudo demonstraram o potencial do SIM para a promoção do uso racional de medicamentos, uma vez que as respostas às SI possibilitaram esclarecimento de questões acerca de uso correto, interações, conservação e reações adversas a medicamentos.

Sobre os outros estudos que subsidiaram essa discussão, houve uma dificuldade em encontrar avaliações de CIM/SIM nacionais. Por isso, foi necessário comparar com trabalhos de outros países. Esta dificuldade eleva a importância deste estudo por contribuir com a discussão sobre os CIM/SIM brasileiros.

\section{CONCLUSÃO}

Avaliando as informações prestadas pelo SIM, depreende-se que o serviço apresentou potencial utilidade na promoção do uso racional de medicamentos, prestando informações independentes e qualificadas, quando solicitado. O perfil de SI e de respostas às SI mostrou ser um tanto específico em relação aos resultados constatados em outros CIM/ SIM. Estes dados servem de subsídio para o aprimoramento do SIM quanto à implantação da informação ativa, no caso, elaboração de boletins informativos, principalmente, baseados nos temas de maior prevalência de SI, além de acompanhamento de impacto das respostas às SI sobre o usuário assistido. Desta forma, pode contribuir tanto para melhor qualificação do corpo profissional, quanto para a melhoria da assistência ao usuário, consequentemente, melhoria da qualidade do serviço de saúde, principalmente, sob a dimensão segurança do paciente.

\section{REFERÊNCIAS}

1 Kornis GEM, Braga MH, Paula PAB. Transformações recentes da indústria farmacêutica: um exame da experiência mundial e brasileira no século XXI. Physis. 2014;24(3):885-908. DOI: 10.1590/S010373312014000300012 .

2 Storpirtis S, Mori ALPM, Yochiy A, Ribeiro E, Porta V. Farmácia clínica e atenção farmacêutica. 1. ed. Rio de Janeiro: Guanabara Koogan. 2011.
3 Escalante-Saavedra PA, Marques-Batista G, Maniero HK, Bedatt-Silva R, Calvo-Barbado DM. Brazilian drug information centre: descriptive study on the quality of information 2010-2015. Farm Hosp. 2017;41(3):334-345. DOI: $10.7399 /$ fh.2017.41.3.10641.

4 OMS. Organização Mundial da Saúde. O papel do farmacêutico no sistema de atenção à saúde: Relatório do Grupo Consultivo da OMS. 1988. 
Bisson MP. Farmácia clínica \& atenção farmacêutica. 2. ed. Barueri: Manole. 2007.

6 Vidotti CCF, Silva EV, Hoefler R. Implantação e desenvolvimento de centro de informação sobre medicamentos em hospital como estratégia para melhorar a farmacoterapia. Pharm. Bras. 2010:1-23.

7 BRASIL. Ministério da Saúde. Portaria no 2.647, de 4 de novembro de 2013. Institui a Rede Brasileira de Centros e Serviços de Informação sobre Medicamentos (REBRACIM). Diário Oficial da União, $n^{\circ} 215,5$ de novembro de 2013. Seção 1. p. 41-2.

8 Nicoletti MA, Marques GRS, Aguiar PM, Storpirtis S. Diagnóstico situacional da atuação dos centros de informações sobre medicamentos no Brasil. Rev. Eletr. Farm. 2017;14(1):5-14.

9 Hoover RM, Hunter ML, Krueger KP. Survey of workload and operational characteristics for academic drug information centers. Curr Pharm Teach Learn. 2018;10(5):579-583. DOI: 10.1016/j. cpt1.2018.02.003.

10 Nunes JO, Escalante-Saavedra PA, Oliveira MFB. Mídias utilizadas por centros de informação sobre medicamentos para promoção do uso racional dessa tecnologia no Brasil. Infarma - Ciênc Farm. 2018;30(Supl 1):435. DOI: 10.14450/23189312.v30.esup1.a2018.pp24-454.

11 WHO. World Health Organization. Anatomical Therapeutic Chemical (ATC): structure and principles. Geneva: WHO; 2013. [citado em 8 de janeiro de 2018]. Disponível em: http://www.whocc.no/atc/structure_and_ principles/.

12 Das SK, Acharya S; Pr AV; Gupta S. Drug information service as pharmaceutical care: provided by Clinical Pharmacists' in a South Indian government hospital. Austin J Pharmacol Ther. 2014;2(6):1-3.

13 Kumar SV, Chakilam V, Pabba A. Performance of drug information centre by a Clinical Pharmacist in a tertiary care teaching hospital, Warangal, Andhra Pradesh, India. IJPPDR. 2013;3(1):1-5.

14 Rajanandh GM, Seenivasan P Ahalya SP, Anjali R. Assessment of pharmacists-led drug information service in a tertiary care hospital in India. J. Med Sci. 2017;17(2):102-106.

15 Peixinho RL, Souza WC, Santos M, Pio IDSL, Nunes DM. Centro de informações sobre medicamentos: do processo de informação à prática do serviço. Infarma Ciênc Farm. 2018;30(Supl 1):358. DOI: 10.14450/23189312.v30.esup1.a2018.pp24-454.
16 Peter AV, Murali A, Tomy T, Londhe SP. Assessment and utilization of drug information services and creating awareness for enhanced utilization of drug information centre in a tertiary care teaching hospital. Asian J Pharm Clin Res. 2017;10(5):270-274.

17 Rapkiewicz JC, Trebien HA, Pereira JG, Lacerda RB, Paula CS. Centro de Informação sobre Medicamentos do Conselho Regional de Farmácia do Paraná (CIM/CRF-PR): avaliação do serviço e satisfação do usuário. Rev. Bras. Farm. 2010;91(3):111-118.

18 Silva VN, Diniz RS, Egito EST, Azevedo PRM, Vidotti $\mathrm{CCF}$, Araújo IB. A way to evaluate the peculiarities of drug information centers in university hospitals. Lat. Am. J. Pharm. 2011;30(5): 902-907.

19 Behera SK, Xavier AS, Gunaseelan V, Ravindra BK, Selvarajan S, Chandrasekaran A, Dkhar SA. Drug information center as referral service in a South Indian tertiary care hospital. Int J Pharm Investig. 2017;7(4):182-187. DOI: 10.4103/jphi.JPHI_90_17.

20 Ashenef A, Reshid E, Yilma Z, Melaku T, Chane T. Assessment of the use and status of new drug information centers in a developing country, Ethiopia: the case of public university hospital drug information centers. BioMed Res Int. 2018:1-11. DOI: 10.1155/2018/3840976.

21 ANVISA. Agência Nacional de Vigilância Sanitária. Bulário eletrônico. [citado em 15 de janeiro de 2019]. Disponível em: http://portal.anvisa.gov.br/bulario-eletronico1.

22 Ali AA, Yusoff SM, Joffry SM, Wahab MSA. Drug information service awareness program and its impact on characteristics of inquiries at DIS Unit in Malaysian public hospital. Arch Pharm Pract. 2013;4(1):9-14. DOI: 10.4103/2045-080X.111576.

23 Reppe LA, Lydersen S, Schjøt J, Damkier P, Christensen HR, Kampmann PJ, Böttiger Y, Spigset O. Relationship between time consumption and quality of responses to drug-related queries: a study from seven drug information centers in Scandinavia. Clin Ther. 2016;38(7):1738-1749. DOI: 10.1016/j.clinthera.2016.05.0100149-2918.

24 Cheng FWT, So SWK, Fung BWT, Hung WH, Lee VWY. Online drug information platform for the public in Hong Kong - review of local drug information use and needs. Int J Med Inform. 2018;114:27-34. DOI: 10.1016/j.ijmedinf.2018.03.006. 